\title{
Curative embolization of iatrogenic inferior mesenteric arteriovenous fistula 14 years after right hemicolectomy
}

\author{
Stavros Charalambous ${ }^{a}$, Andreas Veniamin ${ }^{b}$, Vassilis Valatas ${ }^{b}$, Adam Hatzidakis ${ }^{c}$ \\ University Hospital of Heraklion, Crete; AHEPA University Hospital of Thessaloniki, Greece
}

\begin{abstract}
Inferior mesenteric arteriovenous fistula (IMAVF) is a rare condition with 40 reported cases. It can be of congenital, idiopathic or acquired etiology. Acquired IMAVF occurs after trauma or has an iatrogenic origin due to abdominal interventions, mainly operations involving the left hemi-colon. A new case of iatrogenic IMAVF is described, which became symptomatic 13 years after right hemicolectomy and was diagnosed radiologically one year later. This case was treated successfully by means of endovascular arterial embolization. To our knowledge, this is only the second reported case of acquired IMAVF following right hemicolectomy.
\end{abstract}

Keywords Inferior mesenteric artery, arteriovenous fistula, endovascular embolization, portal hypertension, ischemic colitis

Ann Gastroenterol 2020; 33 (3): 1-4

\section{Introduction}

Arteriovenous fistula (AVF) involving the inferior mesenteric artery (IMA) and vein (IMV) is relatively rare, with 40 published cases in total [1-6] and just 15 of acquired etiology [1,7-9]. Inferior mesenteric arteriovenous fistula (IMAVF) usually manifests with a variety of signs and symptoms resulting from pathophysiologic changes, including abdominal pain, gastrointestinal bleeding, ischemic colitis, and portal hypertension [1]. IMAVF is usually diagnosed radiologically and the treatment strategies are case-specific, involving either endovascular embolization or surgical intervention.

\section{Case report}

Informed consent was obtained from the patient to publish the case details and images. Institutional Review Board approval was not required.

Department of ${ }^{a}$ Medical Imaging, Interventional Radiology Unit, University Hospital of Heraklion, Crete (Stavros Charalambous); ${ }^{\mathrm{b}}$ Gastroenterology Unit, University Hospital of Heraklion, Crete (Andreas Veniamin, Vassilis Valatas); 'Radiology, Interventional Radiology Unit, AHEPA University Hospital of Thessaloniki (Adam Hatzidakis), Greece

Conflict of Interest: None

Correspondence to: Stavros Charalambous, MD, Resident in Radiology, Department of Medical Imaging, University Hospital of Heraklion, Greece, University St., 71500 Stavrakia-Heraklion, Crete, Greece, e-mail: st.charalambous@hotmail.com

Received 4 December 2019; accepted 21 January 2020; published online 14 March 2020

DOI: https://doi.org/10.20524/aog.2020.0463
A 73-year-old female with an 18-month history of left lower abdominal pain and watery diarrhea, presented to us after her symptoms worsened. She reported melena, loss of appetite and unexplained weight loss of $10 \mathrm{~kg}$ during the last 6 months. The patient also reported medically controlled arterial hypertension, chronic obstructive pulmonary disease, and a history of right hemicolectomy 14 years previously for complicated acute appendicitis. On presentation, she was afebrile and hemodynamically stable. Physical examination revealed epigastric pain radiating to the left lower quadrant, without tenderness or abdominal mass, and no bruit or thrills were noticed during abdominal auscultation. Digital rectal examination was negative for melena. Laboratory studies were only significant for anemia (hemoglobin $9.5 \mathrm{~g} / \mathrm{dL}$, albumin $3 \mathrm{~g} / \mathrm{dL}$, C-reactive protein $1.07 \mathrm{mg} / \mathrm{dL}$ ). No other positive findings were noticed and the patient was admitted to the gastroenterology department for further investigation.

Upper gastrointestinal endoscopy showed erythema with telangiectasias on gastric fundus, and signs of severe portal hypertensive gastropathy. Colonoscopy showed edema of the left hemi-colon, a mosaic pattern-like appearance of the colonic mucosa with arachnoid telangiectasias, and edema of the rectum suggesting portal colopathy. Colonic biopsies did not confirm any specific pathological entity. An abdominal Doppler ultrasound examination of the spleno-portal venous system was normal. Abdominal computed tomography angiography (CTA) demonstrated edema and wall thickening of a very long segment of left hemi-colon, extending from the transverse colon to the end of the rectum, with a large fistula between the IMA and IMV. The vessels involved were enlarged and tortuous, with a diameter of $7 \mathrm{~mm}$ at the origin of the IMA and $14 \mathrm{~mm}$ in the most dilated part of IMV. A 3-dimensional scrupulous analysis of volume rendering was used to create a vascular map to display the feeding artery, draining vein and the AVF itself (Fig. 1A). 
Due to worsening of portal hypertension symptoms and portal colopathy, the diagnosis and treatment of IMAVF were facilitated by angiography and subsequent embolization of the AVF sites. Angiography of the IMA confirmed the location of the AVF, so we proceeded to coil embolization in 2 IMA feeding vessels proximal to the fistula bed and a suspicious distal sigmoidal branch, to allow a more distal and controlled embolization (Fig. 1B,C). Final angiography revealed a sufficient result with total occlusion of the AVF, intact internal iliac and mesenteric arteries, and intact perfusion of the sigmoid colon through collaterals (Fig. 1D). After embolization, the patient remained hemodynamically stable, afebrile, with constant levels of hemoglobin and hematocrit (hemoglobin $9.4 \mathrm{~g} / \mathrm{dL}$ ). On the fifth post-interventional day, a new CTA demonstrated extensive wall thickening of the left hemi-colon. The IMAVF appeared with reduced contrast enhancement, but still patent as a result of collateral feeding branches through the right internal iliac (r-IIA), lower rectal arteries and from a sigmoidal artery (Fig. 2A). In view of these findings, a second session of arterial embolization was proposed. Selective angiography of the IMA confirmed the findings (Fig. 2B) and coil embolization was performed in a proximal sigmoidal artery and the right lower rectal branches from the r-IIA. Completion angiography revealed improved AVF occlusion and adequate sigmoid perfusion.
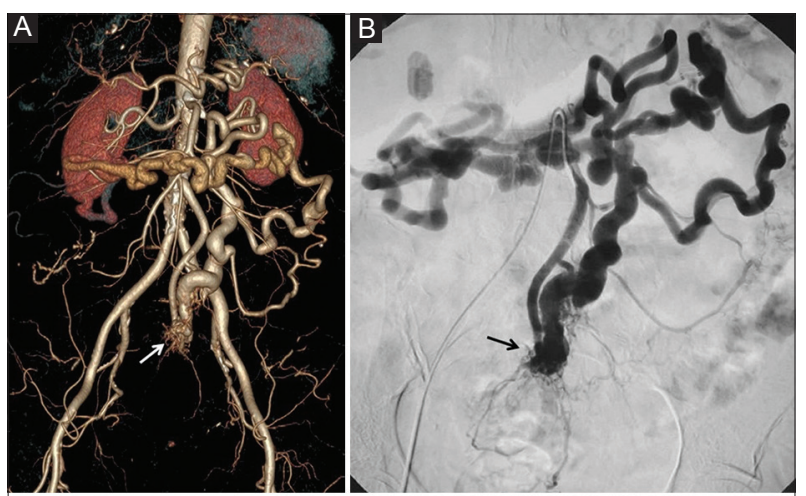

C

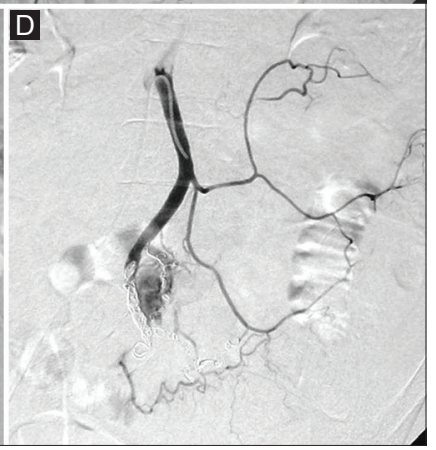

Figure 1 (A) Three-dimensional volume rendering analysis from computed tomography angiography, showing enlarged fistulous communication (white arrow) between the inferior mesenteric artery and vein. (B) Selective angiogram from the inferior mesenteric artery confirms the location of the arteriovenous fistula (arrow). (C) Superselective coil embolization of the inferior mesenteric artery feeding branches (black arrows) and of a suspicious distal sigmoidal feeding branch (white arrow). (D) Completion coil-embolization arteriogram showed almost complete cessation of the flow across the arteriovenous fistula and intact perfusion of the sigmoid colon
Although the patient was discharged in good condition, she continued to have recurrent episodes of abdominal pain and watery diarrhea, so she was readmitted 50 days later. CTA revealed reduced swelling of the colon and contraction of the aberrant vascular branches; however, a small-untreated area of AVF was revealed between the IMA and IMV (Fig. 3). A third session of selective coil embolization was decided upon, and the feeding artery at the AVF junction was successfully occluded. On the final angiography, the vascular abnormality was completely thrombosed, with the rest of the mesenteric vessels remaining intact. Recovery was uneventful, with no diarrhea, recurrence of abdominal pain or signs of gastrointestinal bleeding, and patient was discharged in good general condition

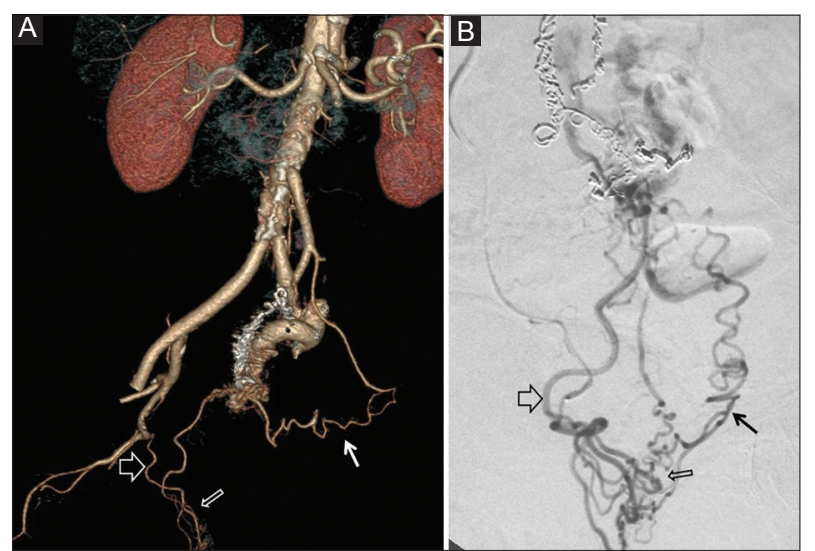

Figure 2 (A) Three-dimensional volume rendering analysis from postoperative computed tomography angiography, showing arteriovenous communication of inferior mesenteric vessels through collaterals from the right internal iliac (open arrow), lower rectal (thin open arrow) and sigmoidal arteries (arrow). (B) Selective angiography from the right internal artery confirms the findings shown in Fig. 2A. (Right internal iliac [open arrow], lower rectal [thin open arrow] and sigmoidal arteries [arrow])

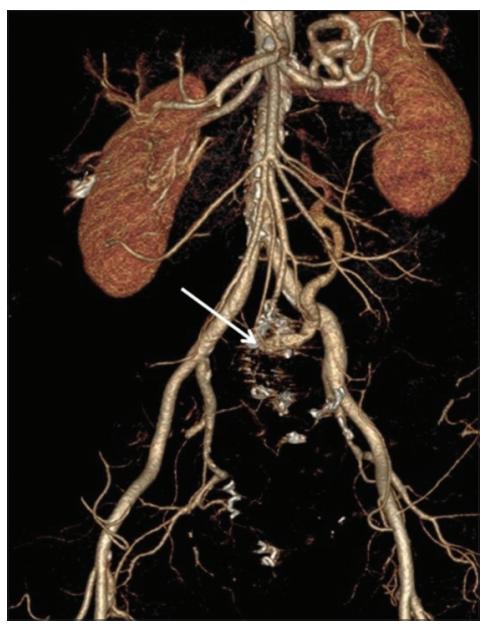

Figure 3 Three-dimensional volume rendering analysis from computed tomography angiography after the second embolization, showing the previous embolization sites without further fistula perfusion and a small arteriovenous malformation between the inferior mesenteric artery and vein (white arrow) 
Table 1 Cases of inferior mesenteric arteriovenous fistula of acquired etiology

\begin{tabular}{|c|c|c|}
\hline Study-Case & Etiology-Previous operation & Management/Treatment \\
\hline Houdard et al, $1970[1,7]$ & Excision of splenic flexure & Open surgery \\
\hline Hirner et al, $1978[1,7,9]$ & Obstetrical operation & Open surgery \\
\hline Capron et al, 1984 [1,2,5-7,9] & Sigmoid volvulus & Endovascular embolization \\
\hline Slutski et al, 1988 [1] & Anterior rectum resection & Endovascular embolization \\
\hline Peer et al, $1989[1,7]$ & Anterior rectum resection & Endovascular embolization \\
\hline Pietri et al, $1990[1,7]$ & Left colectomy & Open surgery \\
\hline Pietri et al, $1990[1,7]$ & Left colectomy & Endovascular embolization \\
\hline Vauthey et al, 1997 [1] & Blunt trauma & Combined therapy ${ }^{*}$ \\
\hline Okada et al, $2002 \quad[1,3,5-7,9,10]$ & Sigmoidectomy & Open surgery \\
\hline Fabre et al, $2005[1,7]$ & Sigmoidectomy & Endovascular embolization \\
\hline Tomoni et al, $2005[4,7]$ & Sigmoidectomy & Open surgery \\
\hline Gorospe et al, 2012 [10] & Right colectomy & Combined therapy* \\
\hline Jeon et al, 2013 & Blunt trauma & Open surgery \\
\hline Carbonell et al, 2014 [8] & Sigmoidectomy & Endovascular embolization \\
\hline Brucher et al, 2014 [9] & Sigmoidectomy & Endovascular embolization \\
\hline Current study 2019 & Right colectomy & Endovascular embolization \\
\hline
\end{tabular}

${ }^{\star}$ Combined therapy=endovascular embolization and open surgery

a few days later. During a 6-month follow up on an outpatient basis, all gastrointestinal symptoms had subsided, hemoglobin returned to normal levels and the patient began to gain weight (hemoglobin $12.3 \mathrm{~g} / \mathrm{dL}$, albumin $3.8 \mathrm{~g} / \mathrm{dL}$ ).

\section{Discussion}

AVF involving the inferior mesenteric vessels is a rare condition with 40 reported cases, including 15 of acquired etiology (Table 1) [1,7-9]. Acquired IMAVF usually occurs after abdominal intervention involving left hemicolectomy, and may be due to transfixion sutures that simultaneously pass through the arteries and veins, or ligation of an artery and vein [1]. The vast majority of patients with IMAVF present with signs and symptoms that result from the pathophysiologic changes of the AVF, which acts as left-to-right shunt: these include abdominal pain and a palpable abdominal mass. The increased blood flow through an IMAVF may cause portal hypertension, and decreased arterial flow to the bowel wall could result in nonocclusive ischemic colitis [1,2]. However, some patients may remain asymptomatic, with only incidental detection of AVF on imaging [3]. Mesenteric AVF is usually diagnosed within days or weeks of the initial injury, but delays in diagnosis of up to 20 years have been reported, as in the present case [6]. CTA remains a fast and easily accessible imaging modality for the initial investigation of a possible mesenteric vascular abnormality. There are 2 potential treatment strategies for IMAVFs: open surgery, or endovascular embolization followed by open surgery in case of failure or complications $[1,2,6]$.
The management of this condition is complex and needs cooperation among the medical team, which includes radiologists/interventional radiologists, gastroenterologists and surgeons. Coil embolization is an effective method that can avoid surgical resection. It is less invasive and potentially safer, with a lower rate of hemorrhage, but carries the risk of organ ischemia, especially if more than one feeding artery is embolized or embolic material passes into the portal circulation [1-3]. We decided to treat our patient more cautiously, with step-by-step embolization in order to prevent ischemia and potential necrosis, resulting surgical colectomy. With this staged strategy, our chances of success were better and we could reassess the clinical result every time before deciding to re-intervene.

\section{References}

1. Athanasiou A, Michalinos A, Alexandrou A, Georgopoulos S, Felekouras E. Inferior mesenteric arteriovenous fistula: case report and world-literature review. World J Gastroenterol 2014;20:82988303.

2. Das Gupta J, Rana MA, Delu A, Guliani S, Langsfeld M, Marek J. Spontaneous inferior mesenteric arteriovenous fistula as a cause of severe portal hypertension and cardiomyopathy. J Vasc Surg Cases Innov Tech 2019;5:113-116.

3. Coulier B, De Wispelaere JF, Bueres I, et al. Acute massive congestive ischaemic colitis related to inferior mesenteric arteriovenous malformation. BJR Case Rep 2016;2:20150275.

4. Faghihi Langroudi T, Shabestari AA, Pourghorban R, Pourghorban R. Idiopathic inferior mesenteric arteriovenous 
fistula: a rare cause of pulsatile abdominal mass. Indian J Surg 2015;77(Suppl 1):S84-S86.

5. Bettenworth D, Rijcken E, Müller KM, Mosch-Messerich A, Heidemann J. Rare cause of upper gastrointestinal bleeding in a 27-year-old male patient. Gut 2012;61:1367.

6. Lee S, Chung J, Byungkwon A, Lee S, Baek S. Inferior mesenteric arteriovenous fistula. Ann Surg Treat Res 2017;93:225-228.

7. Jeon DO, Park JS, Kim JE, et al. [A case of traumatic inferior mesenteric arteriovenous fistula]. Korean J Gastroenterol 2013;62:296-300.
8. Carbonell S, Ortiz S, Enriquez P, Lluis S. Arteriovenous fistula in the inferior mesenteric territory. Cir Esp 2014;95:e23.

9. Brucher N, Moskovitch G, Otal P, Chaufour X, Rousseau H. Inferior mesenteric arteriovenous fistula treated by percutaneous arterial embolization: a breathtaking story! Diagn Interv Imaging 2014;95:85-86.

10. Gorospe E, Leggett C, Sun G. Inferior mesenteric arteriovenous malformation: an unusual case of ischemic colitis. Ann Gastroenterol 2012;25:165. 